\title{
Development and validation of sunlight exposure measurement questionnaire (SEM-Q) for use in adult population residing in Pakistan
}

Quratulain Humayun ${ }^{1}$, Romaina lqbal ${ }^{2}$, Iqbal Azam², Aysha Habib Khan ${ }^{3}$, Amna Rehana Siddiqui ${ }^{2}$ and Naila Baig-Ansari ${ }^{4}$

\begin{abstract}
Background: Vitamin D deficiency has been identified as a major public health problem worldwide. Sunlight is the main source of vitamin $D$ and its measurement using dosimeters is expensive and difficult for use in population-based studies. Hence, the aim of this study was to develop and validate questionnaires to assess sunlight exposure in healthy individuals residing in Karachi, Pakistan.

Methods: Two questionnaires with seven important items for sunlight exposure assessment were developed. Fifty four healthy adults were enrolled based on their reported sunlight exposure (high $=17$, moderate $=18$, low=19) from Aga Khan University, Karachi. Over four days, study participants were asked to wear a dosimeter between sunrise and sunset and report time spent and activities undertaken in the sun for questionnaire validation. Algorithm for item weightage was created as an average score based on ultraviolet B percentage received. Blood samples were obtained for serum vitamin D.

Results: The mean time (minutes) spent in sun over 4 days $( \pm$ SD) was $69.5( \pm 32)$ for low, $83.5( \pm 29.7)$ for moderate and $329( \pm 115)$ for high exposure group. The correlation between average time (minutes) spent in sun over 4 days and mean change in absorbance of UV dosimeters for 4 days was $0.60(p<0.01)$. Correlation between average score and vitamin $D$ levels was found to be $0.36(p=0.01)$ for short term questionnaire score, $0.43(p=0.01)$ for long term questionnaire score in summers and $0.48(p=0.01)$ in winters.
\end{abstract}

Conclusions: The sunlight exposure measurement questionnaires were valid tools for use in large epidemiological studies to quantify sunlight exposure.

Keywords: Vitamin D deficiency, Dosimeters, Validation, Correlation, Sunlight exposure

\section{Background}

In recent years, exposure to sunlight has been a subject of epidemiological interest both due to its beneficial as well as adverse effects on human health. It is an important etiological factor in the development of skin cancers and sun-related eye problems when exposure is high, or Vitamin D Deficiency (VDD) when exposure is low[1,2]. However, the exposure to solar ultraviolet radiation (UVR) is an essential step for the production of Vitamin $\mathrm{D}$, and also the main source of vitamin $\mathrm{D}$ in human

\footnotetext{
* Correspondence: romaina.iqbal@aku.edu

${ }^{2}$ Department of Community Health Sciences, Aga khan University, Stadium road, Karachi, Pakistan

Full list of author information is available at the end of the article
}

body [3,4]. Ultraviolet B (UVB) rays in the solar UVR spectrum produce vitamin $\mathrm{D}$ in the human body. UVB rays penetrate uncovered skin and converts cutaneous 7dehydrocholesterol to pre-vitamin $\mathrm{D}_{3}$, which in turn becomes vitamin $D_{3}[5,6]$. The enteral route is not considered a good source of vitamin D as foods of animal origins e.g. fish, beef, eggs, milk, cheese etc., generally lack the required amount of vitamin D unless fortified [7].

VDD used to be considered rare in those parts of the world that had plenty of sunshine all year round but WHO now estimates that globally one billion people have VDD or insufficiency [1,8]. Geographically, Pakistan lies in a region with adequate sunlight throughout the year [9] with UV index ranging between 5 and 11 during different seasons. However, despite its geographical location, 
the prevalence of VDD is reported to be high, ranging from $20 \%$ to $83 \%$ [10-12].

Previously sunlight exposure has been measured by dosimeters or by a short sunlight diary. However these tools have certain limitations. The dosimeters are prohibitively expensive, therefore cannot be used in large epidemiological studies and the diaries estimate the duration of exposure to sunlight (time in minutes/day) with adjustment either for none or few covariates that could influence UVB activity, such as use of sunscreens, type of clothing, traveling in sun, working in shady area, sun protection practices and skin tone of the individual etc. A major limitation in the conduct of more research in the area of VDD is the lack of appropriate and inexpensive tools for measuring sunlight exposure, which is an important determinant of vitamin D levels in population based studies. As per our knowledge, no questionnaire is currently available for assessing sunlight exposure in Pakistani population. This study aimed to develop and validate a Sunlight Exposure Measurement Questionnaire, for quantification of sunlight exposure in our population.

\section{Methods}

\section{Study setting and design}

This was a validation study and was conducted at the Aga Khan University (AKU) from December 2009 to April 2010, in Karachi, Pakistan. The study population consisted of students, faculty and staff working at AKU, from varied socioeconomic backgrounds living in various parts of Karachi. The study population was selected in three groups according to the perceived sunlight exposure pattern: 1) low sunlight exposure, i.e. those who worked indoors most of the time. This group included faculty and staff; 2) moderate sunlight exposure, this category consisted undergraduate and graduate students; 3) high sunlight exposure, i.e. AKU employees who worked outdoors in the sun most of the day such as security guards, drivers, gardeners and housekeepers. The participants falling in one of the three groups were approached, briefed about the study and those who agreed to participate in the study an informed consent was obtained from them.

\section{Eligibility criteria}

Apparently healthy faculty, staff and students working at AKU were included. Subjects with known skin disorders, immuno-compromised status or a history of using vitamin $\mathrm{D}$ injectables or drugs affecting serum vitamin $D$ status in the past one year were not recruited for the study.

\section{Development of sunlight exposure questionnaires}

We developed two pre-coded sunlight exposure measurement questionnaires (SEM-Q) after detailed and thorough literature search. First questionnaire was long term (LT) with reference period of one year and second questionnaire was short term (ST) with reference period of one day (current day) with a grid divided in one hour intervals from sunrise to sunset. Table 1 shows the comparison of the two questionnaires developed for assessing sunlight exposure.

The LT-SEMQ had three elaborate components, the socio-demographic, sun exposure measurement, and skin tone assessment component.

Both the questionnaires were developed in English, translated into Pakistan's national language, Urdu, and then back-translated into English to check for content validity of the questionnaire.

\section{Pre-testing}

Pre-testing of the questionnaires was also carried out on $15 \%$ of the sample $(n=8)$ prior to the actual data collection in order to ensure standardization and reliability of the questionnaires. The questionnaires were then revised and finalized on the basis of the pretest results.

\section{Factors affecting individual UVR exposure}

The personal and atmospheric factors that affect UVB radiation exposure and vitamin $\mathrm{D}$ synthesis were included in the questionnaire as domains. These included UVR intensity and exposure duration in the sun [13,14], skin tone of the individual [15], use of sunscreens and other cosmetics [16], other sun protection practices (seeking shade under trees/building [17], clothing [18], hats [19], glass/windows [20], cloud cover [21], and occupational behavior[14,22]. Certain other factors that are considered important determinants of an individual's exposure to UVR, such as, atmospheric pollution [23], altitude [24], season [25], surface reflection [26], and ozone [27] were either indirectly catered (surface reflection), were not of primary interest (pollution) in the study or were not applicable to our study setting (altitude, season) and hence not included.

\section{Development of the scoring system for SEM-Q's}

We developed a scoring algorithm for estimation of sunlight exposure (SE) of individuals by taking into consideration all domains that effect $\mathrm{SE}$ at an individual level including time (in minutes) of sun exposure and different domains/mediums that protects from sun exposure e.g. use of sunscreen, standing under the tree, clothes worn/body covered. Table 1 also shows the different domains included in the two questionnaires. For short term, the score was developed for SE over one day while for LT SEM-Q, the scoring was created for a typical one week exposure over the previous 1 year for summer and winter seasons separately. The score was developed by giving different weights (ranging between 0 and 1), 
Table 1 Comparison of Long Term (LT) and Short Term (ST) Sunlight Exposure Measurement Questionnaires (SEM-Q)

\begin{tabular}{|c|c|c|}
\hline & LT SEM-Q* & ST SEM-Q ${ }^{* *}$ \\
\hline Reference period & 1 year: Previous year summer and winter seasons & $\begin{array}{l}1 \text { day: Hourly routine sun exposure recorded } \\
\text { for } 1 \text { day }\end{array}$ \\
\hline Type of questionnaire & Interviewer administered & Self administered (verified) \\
\hline $\begin{array}{l}\text { Domains: Time (minutes) spent outdoors, } \\
\text { weather, clothing, use of sunscreen, } \\
\text { sun protection practices, use of multivitamin } \\
\text { and skin tone. }\end{array}$ & Typical week or day in previous summers and winters. & $\begin{array}{l}\text { Additional domain: Travel in car/bus with } \\
\text { windows up. } \\
\text { In the form of a grid with time } \\
\text { in one hour interval from sunrise to sunset }\end{array}$ \\
\hline
\end{tabular}

* Long term sunlight exposure measurement questionnaire;

** Short term sunlight exposure measurement questionnaire; 4 questionnaires were filled out on the four days that dosimeters were worn.

according to sun exposure (UVB exposure), to all the domains listed in the questionnaire. For example, if the face was not covered, it received 100\% UVB, hence a proportion of 1 was given, whereas, if the face was covered or partially covered it received $0 \%$ and $50 \%$ UVB's translating into a proportion of 0 and 0.5 respectively. Table 2 shows the different weights given to domain and algorithm established for estimation of sunlight exposure score. The final scoring algorithm was created by multiplying the time (minutes) spent in the sun by the proportions of different domains, as provided by Table 2 .

\section{Gold standard for sunlight exposure measurement}

We used Ultraviolet (UV) dosimeters as the gold standard for validating the questionnaires. Dosimeter measures the amount of UVB ionizing radiation (280$315 \mathrm{~nm}$ wavelength) absorbed over a given period of time [24]. The UV dosimeters were purchased and subsequent data analyzed at the University of Southern Queensland (USQ), Queensland Australia. The polysulphone film ultraviolet dosimeters were pinned on the study participant's clothes as a badge. To remain consistent, all study participants were requested to pin the dosimeter on the upper left side of their chest.

\section{Study protocol \& data collection}

Both the questionnaires were administered through a face to face interview. Biochemical assessment was carried out and UV Dosimeter data was collected from each participant. Details of each of these three types of data collection processes are described below.

Participants were administered the LT SEM-Q and at the end of the interview each participant was given four ST SEM-Q and four UV dosimeters, to be used over

Table 2 Algorithm for Estimation of SE Score for Individuals

\begin{tabular}{|c|c|c|}
\hline Variable/item & Percentage given & Explanation \\
\hline \multirow[t]{3}{*}{ Part of the body exposed (clothing) } & 1 if exposed & $1=100 \%$ UVB can penetrate. \\
\hline & 0 if not exposed/covered & \\
\hline & 0.5 if partially covered & \\
\hline \multirow{3}{*}{$\begin{array}{l}\text { Application of sunscreen/cosmetics } \\
\text { on different parts of body (SPF) }\end{array}$} & 1 if no use of any products & $1=100 \%$ UVB \\
\hline & 0.08 if sunscreen and SPF 15 and above & $0.08=8 \%$ UVB penetrates i.e. blocks $92 \%$ \\
\hline & $\begin{array}{l}0.9 \text { if use of other creams and lotions } \\
\text { without knowledge of SPF }\end{array}$ & $0.9=90 \%$ UVB absorption \\
\hline \multirow[t]{2}{*}{ Sun protection practices } & 1 if no protection practices & $1=100 \%$ UVB absorption \\
\hline & 0.4 if used shade of tree/building etc. & $0.4=40 \%$ UVB available for absorption \\
\hline \multirow[t]{3}{*}{ Weather outdoors } & 1 if sunny & $1=100 \%$ UVB on a sunny day \\
\hline & 0.5 if cloudy & $0.5=50 \%$ UVB on a cloudy day \\
\hline & 0.75 if sunny/cloudy & $0.75=75 \%$ UVB if light clouds and sunny \\
\hline \multirow[t]{2}{*}{ Glass windows of car/bus/van } & 0.1 if glass windows up & $\begin{array}{l}0.1=10 \% \text { UVB absorption through glass windows, } \\
90 \% \text { blocked }\end{array}$ \\
\hline & 0.4 if windows down & $0.4=40 \%$ UVB available for absorption into skin \\
\hline \multirow[t]{5}{*}{ Skin tone } & Type $1-0.8$ & $80 \%$ UVB penetration \\
\hline & Type II - 0.675 & $67.5 \%$ UVB penetration \\
\hline & Type III - 0.55 & $55 \%$ UVB penetration \\
\hline & Type IV - 0.425 & 42.5\% UVB penetration \\
\hline & Type V - 0.3 & $30 \%$ UVB penetration by skin \\
\hline
\end{tabular}


4 days. These dosimeters were packed in four individual envelops. Participants were instructed on when and how to wear the dosimeters and to fill the ST SEM-Q at the end of each day that the dosimeter was worn. They were also instructed how to place the used dosimeter back in the respective envelop. The used dosimeters were collected the next day along with the filled ST SEM-Q. The filled ST SEM-Qs were collected after verification, checking for errors and missing information by research assistants for literate participants. The ST SEM-Q was filled the following day for illiterate participants, by the research assistant and same method of interview was employed as that used for verification of ST SEM-Q for literate participants. Interviewer bias and reporting bias were minimized as much as possible by selecting the participants in accordance with the eligibility criteria, by proper training of the research assistants and following the study methodology properly.

Skin tone of the participants was assessed against shade card by matching shade of the skin on the inner side of the forearm of the participant with the shade on the card. This was a component of LT SEM-Q. Shade card of 20 scales was developed with professional help from experts in designing and printing, to match the skin tones of Asian population. Figure 1 shows the recruitment and flow of participants in the study.

Blood sample, for assessing Vitamin D levels, was obtained from the study participants from December
2009 to April 2010, by trained phlebotomist under sterile conditions. Vitamin D status was determined by measuring serum $25 \mathrm{OHD}_{3}$ concentrations by Electrochemiluminescence immunoassay on Elecsys auto analyzer (Roche Diagnostics, USA). For quality control low, medium and high Elecsys Preci Controls were used. The within-run CVs were $5.7 \%, 5.7 \%$, and $5.4 \%$ at concentrations of 25.2 , 39.9 , and $65.6 \mathrm{ng} / \mathrm{ml}$.

Optimal vitamin D levels were taken as 30-100 ng/ml while levels of $21-29 \mathrm{ng} / \mathrm{ml}$ as vitamin D insufficiency and $<20 \mathrm{ng} / \mathrm{ml}$ as vitamin D deficiency [28]. Vitamin D analysis was done in the laboratory towards the end of the data collection and participants with VDD or insufficiency were informed and educated about sun exposure and vitamin D supplements were provided to the study participants.

\section{Statistical analysis}

For descriptive analysis, means and standard deviations were reported for continuous variables, such as, age, time spent in sun, dosimeter readings; frequencies and percentages were reported for nominal and ordinal variables like VD levels, mean and SD was reported for this variable. For inferential analysis, Kruskal Wallis test was used to detect the difference in sunlight exposure, vitamin D levels and time spent in the sun, among the three sunlight exposure groups. Spearman's rank correlation and Pearson's correlation were also carried-out for the

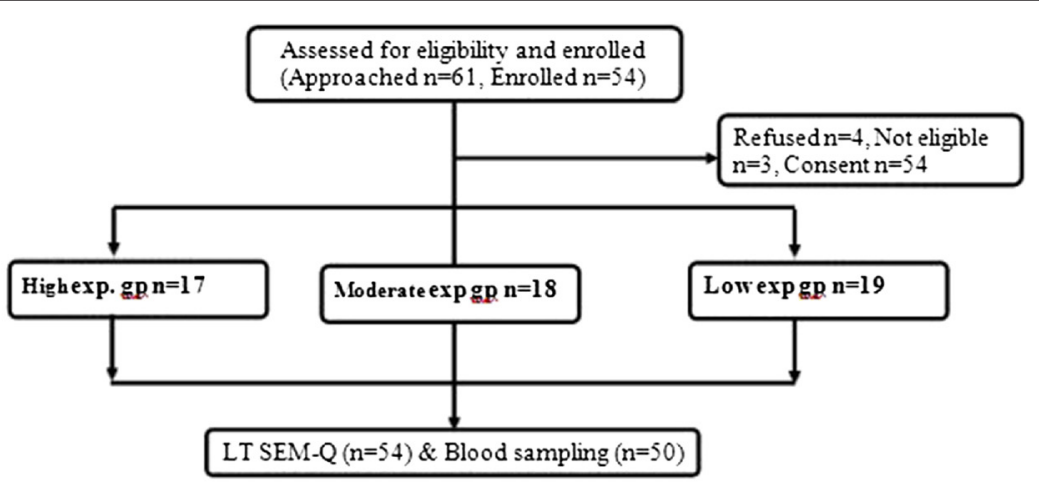

ST SEM-Q (for 4 days)
UV Dosimeters (for 4 days)

Total ST SEM-Q=216

Dosimeters used $n=22$

(Lost 7 dosimeters)

\begin{tabular}{|c|c|c|c|c|}
\hline $\begin{array}{l}\text { Dayl: Dosimeter } \\
\text { \&ST SEM-Q }\end{array}$ & $\begin{array}{c}\text { Day2: Dosimeters } \\
\dot{\&} \text { ST SEM-Q } \\
\text { for day } 1 \text { collected }\end{array}$ & $\begin{array}{c}\text { Day3: Dosimeters } \\
\dot{\&} \text { ST SEM-Q } \\
\text { for day } 2 \text { collected }\end{array}$ & $\begin{array}{l}\text { Day4: Dosimeters } \\
\text { \& ST SEM-Q } \\
\text { for day } 3 \text { collected }\end{array}$ & $\begin{array}{c}\text { Day5: Dosimeters } \\
\dot{\&} \text { ST SEM-Q } \\
\text { for day } 4 \text { collected }\end{array}$ \\
\hline
\end{tabular}

Figure 1 Recruitment and flow of participants in the study. Eligible individuals were recruited into the study into three exposure group's i.e. high, moderate and low exposure, after obtaining informed consent. Participants were administered the LT SEM-Q and at the end of the interview each participant was given four ST SEM-Q and four UV dosimeters, to be used over 4 days. Participants were instructed on when and how to wear the dosimeters and to fill the ST SEM-Q at the end of each day. The used dosimeter and filled ST SEM-Qs was collected the next day after verifying, checking for errors and missing information by research assistants for majority of the participants. 
association between sun exposure scores \& Ultraviolet (UV) dosimeter readings, average scores of SEM-Qs \& vitamin D levels and average scores of LT \& ST SEMQs, respectively. The analysis was carried out using Statistical Package for Social Sciences (SPSS) version 16.0.

\section{Potential confounders and sources of bias}

Potential confounders, such as the outdoor weather, sun protection practices, use of sunscreen and other products, skin tone, and body parts exposed to the sun during the days the dosimeter was worn, were adjusted while creating the scoring for the short term questionnaire.

\section{Ethical approval}

This study protocol was ethically approved by AKU Ethical Review Committee (ERC) on December 4, 2009 (1257-CHS/ERC-09).

\section{Results}

A total of fifty four participants were enrolled in the study. Among the three exposure groups taken, low exposure group accounted for $35.2 \%$ of the total sample $(\mathrm{n}=19)$, moderate for $33.2 \%(\mathrm{n}=18)$ and high exposure group for $31.5 \%(n=17)$. Male participants constituted $53.7 \%$ of the sample. $83.3 \%$ of the total participants were literate with $79.6 \%$ of the study participants having 10 years or more of formal education. Predominant language spoken was Urdu followed by Punjabi. Muslims were $75.9 \%$ of the study participants. Table 3 shows the descriptive analysis of the study participants.

Serum 25(OH) vitamin $\mathrm{D}_{3}$ levels were assessed for 50 study participants. $98 \%(n=49)$ of the participants were either vitamin $\mathrm{D}$ deficient or insufficient. The mean vitamin D level (SD) of our study participants was $12 \mathrm{ng} / \mathrm{ml}$ (5.9), with levels of 9.8(4.7), 11(4.6) and 17(6.5) for low, moderate and high SE groups respectively. Table 4

Table 3 Demographic Characteristics of Study Participants by Sunlight Exposure Group

\begin{tabular}{|c|c|c|c|c|}
\hline \multirow[t]{2}{*}{ Variables } & \multicolumn{3}{|c|}{ Sunlight exposure groups } & \multirow{2}{*}{$\begin{array}{c}\text { Total } \\
(n=54)\end{array}$} \\
\hline & $\begin{array}{c}\text { Low } \\
(n=19)\end{array}$ & $\begin{array}{c}\text { Moderate } \\
(n=18)\end{array}$ & $\begin{array}{c}\text { High } \\
(n=17)\end{array}$ & \\
\hline Age, yrs $\left[\right.$ Mean $\left.\left(S D^{*}\right)\right]$ & $33(9)$ & $31(7)$ & $41(11)$ & $35(10)$ \\
\hline \multicolumn{5}{|l|}{$\operatorname{Sex}[n(\%)]$} \\
\hline Male & $6(31.6)$ & $7(38.9)$ & $16(94.1)$ & $29(53.7)$ \\
\hline Female & $13(68.4)$ & $11(61.1)$ & $1(5.8)$ & $25(46.3)$ \\
\hline \multicolumn{5}{|l|}{ Literacy rate [n (\%)] } \\
\hline Illiterate & 0 & 0 & $9(52.9)$ & $9(16.7)$ \\
\hline Literate & 19 (100) & 18 (100) & $8(47.1)$ & $45(83.3)$ \\
\hline
\end{tabular}

* Standard deviation. shows the distribution of main variables according to the three SE groups.

The correlation coefficient between average time (minutes) spent outdoors over the four days for ST SEM-Q, and average readings of UV dosimeter over four days was $0.60(P<0.01)$. Spearman's rank correlation between average score of ST SEM-Q and serum vitamin D levels was found to be $0.36(P=0.01)$, while Pearson's correlation between serum vitamin D levels and average LT SEM-Q score in summers was $0.43(P=0.01)$ and 0.48 $(P=0.01)$ in winters. Table 5 shows the correlations among the main variables of interest.

Correlation between LT and ST questionnaires was assessed. It was found that the correlation coefficient between average time (minutes) spent outdoors as captured by LT and ST was $0.85(P<0.01)$. Similarly, the correlation coefficient between the scores for LT and ST SEM-Q was observed to be $0.82(P<0.01)$.

\section{Discussion}

We found a good correlation between our ST SEM-Q and dosimeter readings $\left(\mathrm{r}_{\mathrm{s}}=0.60(\mathrm{p}<0.01)\right)$ and fair correlation of $0.36(P=0.01)$ between ST SEM-Q and serum vitamin D levels. Similarly, a good correlation of 0.58 $(P=0.01)$ and $0.60 \quad(P=0.01)$ was observed between dosimeter readings and LT SEM-Q summer and winters respectively. The correlation between vitamin $\mathrm{D}$ levels and LT SEM-Q score for previous year summer and winter season was found to be $0.43(P=0.01)$ and 0.48 $(P=0.01)$ respectively.

Through recent literature search, this study is the first of its kind in South Asian region to develop a questionnaire to assess long and short-term sunlight exposure and validate it against a gold standard objective measure using dosimeter badges. It is also the first study to assess the correlation between the SEM-Q and serum vitamin D levels in Pakistan. Our research strength is that we developed the questionnaire based on the factors that are of importance in a non-western society. It is also a culturally sensitive and acceptable questionnaire in our setting.

The correlation we found between our ST-SEMQ questionnaire and the dosimeter readings $\left(\mathrm{r}_{\mathrm{s}}=0.60 ; P\right.$ $<0.01$ ) were higher than the Australian pilot study, which compared the objective measurement and selfreported ultraviolet radiation exposure during outdoor activities among children and mother pairs and life guards $(r=0.32)$ [29]. The main Australian study later showed fair to good correlations between the sunlight diaries and dosimeters for lifeguards $(r=0.38-0.57)$, parents $(r=0.28-0.29)$ and children $(r=0.18-0.34)$ and these findings were still lower than our study findings [30]. Similarly, our results were higher than previously published data on mothers and children [31], school 
Table 4 Description of Main Variables of Interest

\begin{tabular}{|c|c|c|c|c|}
\hline \multirow[t]{2}{*}{ Variables } & \multicolumn{3}{|c|}{ Sunlight Exposure groups } & \multirow[t]{2}{*}{ Total $(n=54)$} \\
\hline & Low $(n=19)$ & Moderate $(n=18)$ & High $(n=17)$ & \\
\hline 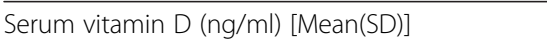 & $9.8(4.7)$ & $11.1(4.6)$ & $17.0(6.5)$ & $12.3(5.9)$ \\
\hline PS UV dosimeter (MED) [Median(IQR)] & $0.5(0.1-10.2)$ & $0.8(0.2-8.2)$ & $3.6(2.1-11.9)$ & $1.2(0.1-11.9)$ \\
\hline \multicolumn{5}{|l|}{ ST SEM-Q* } \\
\hline Time (minutes) spent outdoors & $69.5(32.0)$ & $83.5(29.7)$ & $329(115.7)$ & $95.6(18.7-487.5)^{* * *}$ \\
\hline Adjusted time (minutes) ${ }^{* * *}$ & $43.0(22.5)$ & $64.9(21.8)$ & $258(114.7)$ & $76.3(12.0-463.8)^{* *}$ \\
\hline \multicolumn{5}{|l|}{ LT SEM-Q } \\
\hline Time (minutes) outdoors in summer [Mean(SD)] & $54.5(30.0)$ & $81(62.7)$ & $331.2(63.8)$ & $150(134.9)$ \\
\hline Mean (SD) time outdoors in winters & $59.7(32.5)$ & $89.4(65.0)$ & $310(85.0)$ & $148(127.6)$ \\
\hline
\end{tabular}

$\mathrm{SD}=$ Standard deviation; MED=Minimal erythemal dose; $\mathrm{IQR}=$ Inter quartile range;
${ }^{*}$ Significantly different on Kruskal Wallis test $(P<0.001) ;{ }^{* *}$ Medians (IQR) reported; ${ }^{* * *}$ adjusted for weather outdoors, seeking shade \& travel in vehicle.

children [32] and volunteers recruited from recreational organizations [25]. A possible reason for better correlation of our questionnaire could be the comprehensive nature of the questionnaire and inclusion of items relevant for our population. Moreover, our study population consisted of adults only, working for a university, which could be a reason for better response. Also, most of the studies done previously had included factors in relation to skin cancer development and had taken the time of day when the ultraviolet radiations are the most intense (11 am to $3 \mathrm{pm}$ ) as reference period. We included larger time of sun exposure, which most of the other studies had not taken into consideration.

The results of our study were comparable to some of the other studies that compared personal UVR exposures using PS dosimeters and diary or questionnaire entries among adult volunteers $(r=0.69)$ [33] and school children $(r=0.68)$ [34].

Our correlation between serum Vitamin D and average score for ST SEM-Q was found to be $0.36(P=0.016)$,
$0.43(P=0.01)$ for LT SEM-Q score in summers and 0.48 $(P=0.01)$ for LT SEM-Q score in winters. We did not come across any study that developed scoring for sun exposure questionnaires to assess the correlation between the scores and vitamin $\mathrm{D}$ levels hence we are unable to compare our results with any other study. One study that did measure Vitamin D levels and related it to UVR behavior, correlated the mean daily hours measured by dosimeter in September and Feburary $(r=0.64$, $P=0.001)$ and $(\mathrm{r}=0.53 ; P=0.007)$ [35], however, the study did not look into correlation between Vitamin D and scores for sunlight diaries or questionnaires.

There were a few limitations to our study. The fair correlations of $0.34,0.43$ and 0.48 observed between the serum vitamin D levels and scores for SEM-Qs were probably because of the smaller sample size and hence the inability to find a variation in terms of vitamin $\mathrm{D}$ levels, as majority of study sample was vitamin D insufficient or deficient. Even though our estimated sample size was larger $(n=203)$, due to budget constraints and

Table 5 Correlations between Questionnaires and Dosimeter Readings, and Serum Vitamin D Levels

\begin{tabular}{|c|c|c|c|}
\hline & & Spearman's Rank Correlation & $P$-value \\
\hline \multicolumn{4}{|l|}{ ST SEM-Q } \\
\hline Average time (minutes) spent in sun & UV Dosimeter readings (MED) & 0.601 & $<0.001$ \\
\hline Adjusted-average time (minutes) ${ }^{*}$ & UV Dosimeter readings (MED) & 0.534 & $<0.001$ \\
\hline ST SEM-Q as a score & Serum vitamin D levels & 0.363 & 0.01 \\
\hline \multicolumn{4}{|l|}{ LT SEM-Q } \\
\hline Time (minutes) spent in sun in summers & UV Dosimeter readings (MED) & 0.582 & 0.01 \\
\hline Time (minutes) spent in sun in winters & UV Dosimeter readings (MED) & 0.613 & 0.01 \\
\hline Score of sun exposure in summers (per day) & Serum vitamin D levels & $0.429^{* *}$ & 0.01 \\
\hline Score of sun exposure in Winters (per day) & Serum vitamin D levels & $0.483^{* *}$ & 0.01 \\
\hline
\end{tabular}

ST SEM-Q=Short term sunlight exposure measurement questionnaire; MED=minimual erythemal dose, LT SEM-Q= Long term sunlight exposure measurement questionnaire.

${ }^{*}$ adjusted for sun protection practices, weather and travelling in vehicle; ${ }^{* *}$ Pearson's Correlation reported. 
time limitations, the study was conducted on approximately $25 \%$ of the sample size. We expect that with the larger sample, we would have achieved a larger variation in terms of vitamin D levels and probably a better correlation with the computed score. However, the sample size of 54 with a post hoc power of $83 \%$ was sufficient for meeting the main objective of our study.

We developed and validated two questionnaires i.e. ST and LT SEM-Q, in order to measure and assess both short term sunlight exposure and long term SE over previous 1 year period. Our study assessed sunlight exposure in context with Vitamin D levels, unlike the recent other studies that have assessed sunlight exposure in relation to skin cancer [36]. Both the questionnaires showed good correlation with dosimteres and fair correlations with serum vitamin D levels and, therefore, we can say that both the questionnaires can be used to assess the SE in adults. However, LT SEM-Q might be preferred due to convenience of its use and reflection of a longer duration of sun light exposure inadequacy which may be more relevant for epidemiological work.

The UV dosimeters, although gold standard for measuring UVR, have a few limitations which makes the questionnaires preferred source for assessing sunlight exposure. UV dosimeters are expensive tools and are not readily available in developing countries and hence need to be imported for use. Secondly, the calibration and reading of dosimeters is a complex process and require sensitive equipment and expertise, which is not easily available especially in our settings. Lastly, the dosimeters measure the current exposure to sunlight and do not take into account the long term exposure to sunlight, which is an important factor for vitamin D levels in the body.

\section{Conclusions}

The main outcome of this study was development and validation of a culturally-appropriate and inexpensive tool for assessing sunlight exposure. We found a high correlation between self-reported sun exposure using our tool and the objective measure of sun exposure using the dosimeters and, therefore, SEM-Qs can be used in population based studies for assessing SE. This has great epidemiological value for work in the related vitamin D sunlight exposure measurement and for assessing $\mathrm{SE}$ in relation with other disease outcomes such as skin cancers.

Furthermore, this is the first study in our region for assessing the exposure to sunlight through a questionnaire, and which incorporates methods for scoring based on the factors that affect the UVB rays absorption in the body, factors that may directly affect the amount of previtamin $\mathrm{D}_{3}$ synthesis in the skin.

\section{Competing interests}

The authors declare that they have no competing interests.

\section{Acknowledgements}

The authors are deeply grateful for the detailed support and guidance from Prof Alfio Parisi, this study could not have been possible without his expert and thorough input on working of the instruments used in the study. The authors would also like to acknowledge support by Javeria Rauf, Dr. Kabir Ahmad, Dr. Farhana Shahid, Shamsa Kanwal, Ayesha Khaliq, Mohammad Talah Ayub, Bilal Hussain, and Adnan Saleem for their help at various stages of conducting the study and Zaman Armaghan for his expert skills in developing the skin tone shade cards.

\section{Author details}

${ }^{1}$ Public Health Solutions Pakistan, House No. 578, Street No. 53, G-9/1, Islamabad, Pakistan. 'Department of Community Health Sciences, Aga khan University, Stadium road, Karachi, Pakistan. ${ }^{3}$ Department of Pathology \& Microbiology, Aga Khan University, Stadium road, Karachi, Pakistan. ${ }^{4}$ Indus Hospital, Clinical Research Unit, Korangi Crossing, Karachi, Pakistan.

\section{Authors' contributions}

$\mathrm{QH}$ participated in study design, tool and methods development and carried out data collection, analysis and write up. Rl conceived the idea and supervised every stage of the study. ARS participated in materials and methods development and in technical review of the project. IA participated in the statistical analysis and tool development. AHK and NBA helped to draft the manuscript and gave important inputs. All authors reviewed the manuscript and approved the final version.

Received: 23 December 2011 Accepted: 8 June 2012

Published: 8 June 2012

\section{References}

1. Holick MF, Chen TC: Vitamin D deficiency: a worldwide problem with health consequences. Am J Clin Nutr 2008, 87:1080-1086.

2. Brash DE: Sunlight and the onset of skin cancer. Trends Genet 1997, 13:410-414.

3. Beadle PC: Sunlight, ozone and vitamin D. Br J Dermatol 1977, 97:585-591.

4. Holick MF: Deficiency of sunlight and vitamin D. BMJ 2008, 336:1318-1319.

5. Mullin GE, Dobs A: Vitamin d and its role in cancer and immunity: a prescription for sunlight. Nutr Clin Pract 2007, 22:305-322.

6. Wharton B, Bishop N: Rickets. Lancet 2003, 362:1389-1400.

7. Calvo MS, Whiting SJ, Barton CN: Vitamin D fortification in the United States and Canada: current status and data needs. Am J Clin Nutr 2004, 80:1710S-1716S.

8. Ultraviolet radiation and human health. Fact sheet $N^{\circ} 305$. http://www.who. int/mediacentre/factsheets/fs305/en/.

9. Badruddin SH, Baig-Ansari N: Overview of Vitamin D and its role in Tuberculosis Prevention and Treatment. Infect Dis J Pakistan 2008, 17:135-138.

10. Habib A, lqbal R: Vitamin D Deficiency in an Ample Sunlight Country. JCPSP 2009, 19:267-268.

11. Zuberi LM, Habib A, Haque N, Jabbar A: Vitamin D Deficiency in ambulatory patients. J Pak Med Assoc 2008, 58:482-484.

12. Zargar AH, Ahmad S, Masoodi SR, Wani Al, Bashir MI, Laway BA, Shah ZA Vitamin D status in apparently healthy adults in Kashmir Valley of Indian subcontinent. Postgrad Med J 2007, 83:713-716.

13. Diffey BL: Ultraviolet radiation and human health. Clin Dermatol 1998, 16:83-89.

14. Kimlin MG, Parisi AV, Wong JC: Quantification of personal solar UV exposure of outdoor workers, indoor workers and adolescents at two locations in Southeast Queensland. Photodermatol Photoimmunol Photomed 1998, 14:7-11.

15. Clemens TL, Adams JS, Henderson SL, Holick MF: Increased skin pigment reduces the capacity of skin to synthesise vitamin D3. Lancet 1982, 1:74-76.

16. Matsuoka LY, Ide L, Wortsman J, MacLaughlin JA, Holick MF: Sunscreens suppress cutaneous vitamin D3 synthesis. J Clin Endocrinol Metab 1987 64:1165-1168

17. Diffey BL, Diffey JL: Sun protection with trees. Br J Dermatol 2002, 147:397-399. 
18. Diffey BL: Sun protection with clothing. Br J Dermatol 2001, 144:449-450

19. Diffey BL, Cheeseman J: Sun protection with hats. Br J Dermatol 1992, 127:10-12.

20. Holick MF: Photobiology of vitamin D. In vitamin D, Volume I. secondth edition. Edited by Feldman DPJ, Glorieux FH. Burlington, MA: Elsevier; 2005.

21. Diffey BL, Larko O: Clinical climatology. Photodermatol 1984, 1:30-37.

22. Parisi AV, Meldrum LR, Kimlin MG, Wong JC, Aitken J, Mainstone JS: Evaluation of differences in ultraviolet exposure during weekend and weekday activities. Phys Med Biol 2000, 45:2253-2262.

23. Diffey BL: Sources and measurement of ultraviolet radiation. Methods 2002, 28:4-13.

24. Diffey B: Personal ultraviolet radiation dosimetry with polysulphone film badges. Photodermatol 1984, 1:151-157.

25. Herlihy E, Gies PH, Roy CR, Jones M: Personal dosimetry of solar UV radiation for different outdoor activities. Photochem Photobiol 1994, 60:288-294.

26. Kromann $\mathrm{N}$, Wulf $\mathrm{HC}$, Eriksen $\mathrm{P}$, Brodthagen $\mathrm{H}$ : Relative ultraviolet spectral intensity of direct solar radiation, sky radiation and surface reflections. Relative contribution of natural sources to the outdoor UV irradiation of man. Photodermatol 1986, 3:73-82.

27. Matsui MS, DeLeo VA: Longwave ultraviolet radiation and promotion of skin cancer. Cancer Cells 1991, 3:8-12.

28. Hollis BW, Wagner CL: Normal serum vitamin D levels. N Engl J Med 2005, 352:515-516. author reply 515-516.

29. O'Riordan DL, Glanz K, Gies P, Elliott T: A pilot study of the validity of selfreported ultraviolet radiation exposure and sun protection practices among lifeguards, parents and children. Photochem Photobio/ 2008, 84:774-778.

30. Glanz K, Gies P, O'Riordan DL, Elliott T, Nehl E, McCarty F, Davis E: Validity of self-reported solar UVR exposure compared with objectively measured UVR exposure. Cancer Epidemiol Biomarkers Prev 2010, 19:3005-3012.

31. O'Riordan DL, Stanton WR, Eyeson-Annan M, Gies P, Roy C: Correlations between reported and measured ultraviolet radiation exposure of mothers and young children. Photochem Photobiol 2000, 71:60-64.

32. Dwyer T, Blizzard L, Gies PH, Ashbolt R, Roy C: Assessment of habitual sun exposure in adolescents via questionnaire-a comparison with objective measurement using polysulphone badges. Melanoma Res 1996, 6:231-239.

33. Chodick G, Kleinerman RA, Linet MS, Fears T, et al: Agreement between diary records of time spent outdoors and personal ultraviolet radiation dose measurements. Photochem Photobiol 2008, 84:713-718.

34. Gies P, Roy C, Toomey S, MacLennan R, Watson M: Solar UVR exposures of primary school children at three locations in Queensland. Photochem Photobiol 1998, 68:78-83.

35. Thieden E, Philipsen PA, Heydenreich J, Wulf HC: Vitamin D level in summer and winter related to measured UVR exposure and behavior. Photochem Photobiol 2009, 85:1480-1484.

36. Saridi M, Pappa V, Kyriazis I, Toska A, Giolis A, Liachapoulou A, Skliros E, Birbas K: Knowledge and attitudes to sun exposure among adolescents in Korinthos, Greece. Rural Remote Heal 2009, 9:1162.

doi:10.1186/1471-2458-12-421

Cite this article as: Humayun et al.: Development and validation of sunlight exposure measurement questionnaire (SEM-Q) for use in adult population residing in Pakistan. BMC Public Health 2012 12:421.

\section{Submit your next manuscript to BioMed Central and take full advantage of:}

- Convenient online submission

- Thorough peer review

- No space constraints or color figure charges

- Immediate publication on acceptance

- Inclusion in PubMed, CAS, Scopus and Google Scholar

- Research which is freely available for redistribution 